\title{
Long-Term Functional Effects of Medulloblastoma Treatments
}

Serena Khiantani, MOT/S; Pamela Ponce, MOT/S; Bailey Diprima, MOT/S; Sarika Maymoundok, MOT/S; Leah Murray, MOT/S Faculty Advisor: Anita Witt Mitchell, PHD, OTR, FAOTA Community Practitioner: Heather Clabo, OTR/L

University of Tennessee Health Science Center
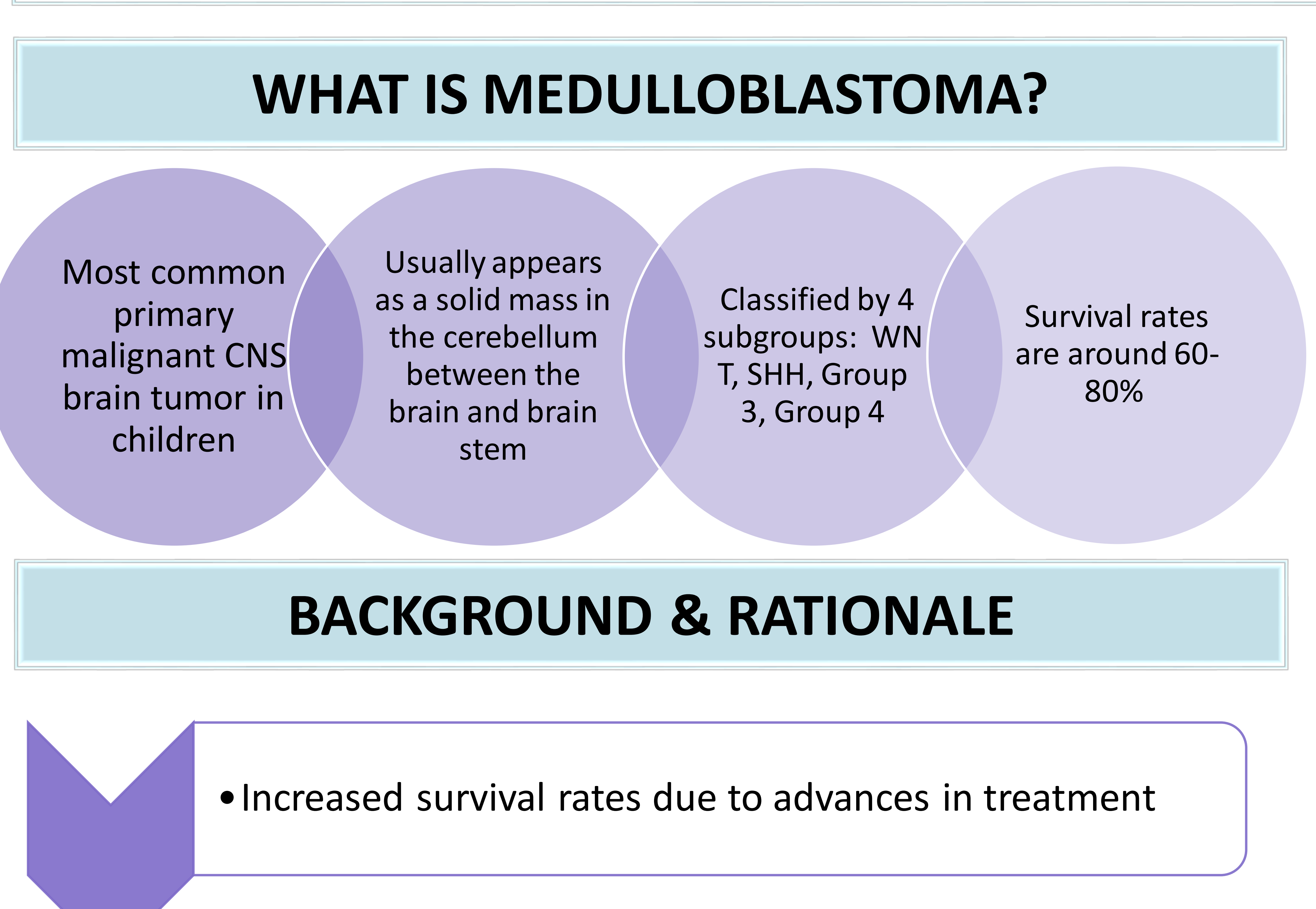

- Survivors are at risk for lasting effects that may impact everyday functional performance and quality of life

- Knowledge of long-term effects could guide client/caregiver education and preventative approaches in occupational therapy

\section{PICO QUESTION \& ELEMENTS}

In children ages 4-18, what are the long-term functional effects of medulloblastoma treatments?

\begin{tabular}{|c|c|}
\hline PICO QUESTION & EXAMPLE SEARCH TERMS USED \\
\hline $\begin{array}{c}\text { Population: } \\
\text { Children ages 4-18 years old }\end{array}$ & $\begin{array}{l}\text { Child, youth, adolescent, teen, } \\
\text { preschool, medulloblastoma }\end{array}$ \\
$\begin{array}{c}\text { Intervention: } \\
\text { Medulloblastoma treatment }\end{array}$ & $\begin{array}{l}\text { Treatment, radiation, surgery, } \\
\text { chemotherapy, tumor resection, } \\
\text { procedure, medication, therapy }\end{array}$ \\
$\begin{array}{c}\text { Outcome: } \\
\text { Functional deficits }\end{array}$ & $\begin{array}{l}\text { Outcome, effect, consequence, } \\
\text { result, product, reaction, functional }\end{array}$
\end{tabular}

\section{SEARCH METHODOLOGY}

Databases:
- Scopus, Cochrane Library, CINAHL, PubMed, Ovid MEDLINE
Inclusion Criteria
- Adult medulloblastoma survivors who received treatment between the ages 4-
18
- Focused on functional effects and/or quality of life
- Explored effects that may connect to functional performance
Exclusion Criteria
- Clients developed secondary disease(s) as a result of the diagnosis and/or
treatment received
- Published more than ten years ago
- Single case studies

\section{MAIN FINDINGS \& LIMITATIONS}

\begin{tabular}{|c|c|c|c|}
\hline $\begin{array}{l}\text { LEVEL OF } \\
\text { EVIDENCE }\end{array}$ & $\begin{array}{c}\text { STUDY AND } \\
\text { QUALITY SCORE }\end{array}$ & MAIN FINDINGS & LIMITATIONS \\
\hline ॥ & $\begin{array}{l}\text { - Moxon-Emre } \\
\text { et al., 2016 } \\
\text { Quality } \\
\text { Score: } 77 \% \\
\text { - Brinkman et } \\
\text { al., 2016 } \\
\text { Quality Score: } \\
77 \% \\
\text { - Szentes et al., } \\
2018 \text { Quality } \\
\text { Score: } 95 \%\end{array}$ & $\begin{array}{l}\text { - Limiting radiation exposure } \\
\text { produces } \uparrow \text { intellectual } \\
\text { outcomes (Moxon-Emre et al., 2016; } \\
\text { Szentes et al., 2018) } \\
\text { - } \downarrow \text { in processing speed, attention, } \\
\text { working memory, and verbal } \\
\text { skills (Moxon-Emre et al., 2016; } \\
\text { Szentes et al., 2018) } \\
\text { - } \text { in generalized and separation } \\
\text { anxiety (Szentes et al., 2018) }\end{array}$ & - Small sample \\
\hline III & $\begin{array}{l}\text { - Edelstein et } \\
\text { al., } \\
2011 \text { Quality } \\
\text { Score: } 77 \%\end{array}$ & $\begin{array}{l}\downarrow \text { in working memory regardless of } \\
\text { age of treatment } \\
\text { Cognitive and physical signs of early } \\
\text { aging regardless of age of diagnosis } \\
\text { - Younger age of diagnosis associated } \\
\text { with a } \downarrow \text { in IQ and academic scores }\end{array}$ & $\begin{array}{l}\text { Small sample } \\
\text { Retrospective } \\
\text { study } \\
\text { Only focuses on } \\
\text { radiation } \\
\text { treatment }\end{array}$ \\
\hline IV & $\begin{array}{l}\text { - Kennedy et al., } \\
2014 \text { Quality } \\
\text { Score: } 86 \% \\
\text { - Saury } \\
\text { \& Emanuelson } \\
\text {,2010 Quality } \\
\text { Score: } 90 \%\end{array}$ & $\begin{array}{l}\downarrow \text { in cognition (e.g., executive } \\
\text { function, attention capacity, } \\
\text { processing speed) }\end{array}$ & $\begin{array}{l}\text { - Not in the US } \\
\text { - No true control }\end{array}$ \\
\hline
\end{tabular}

\section{CLINICAL BOTTOM LINES}

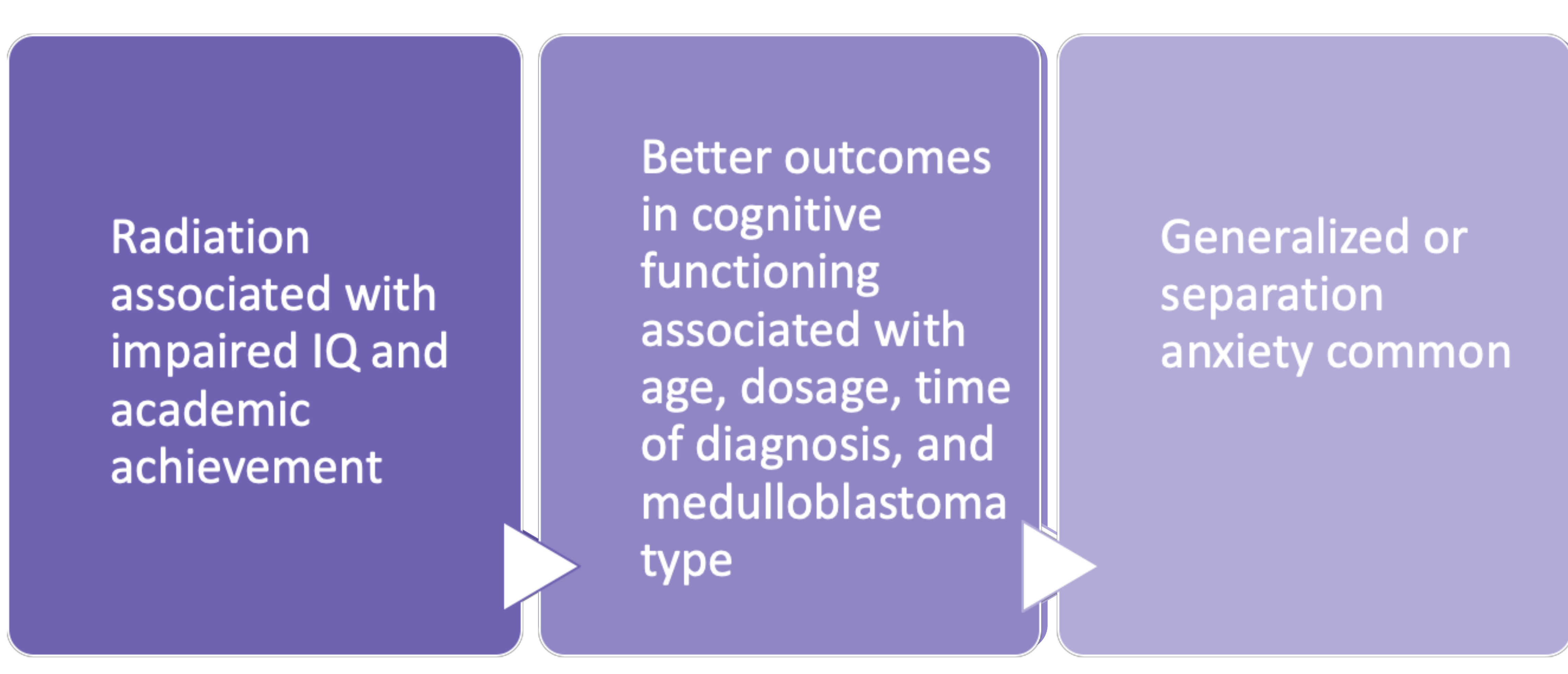

\section{IMPLICATIONS FOR OT PRACTICE}

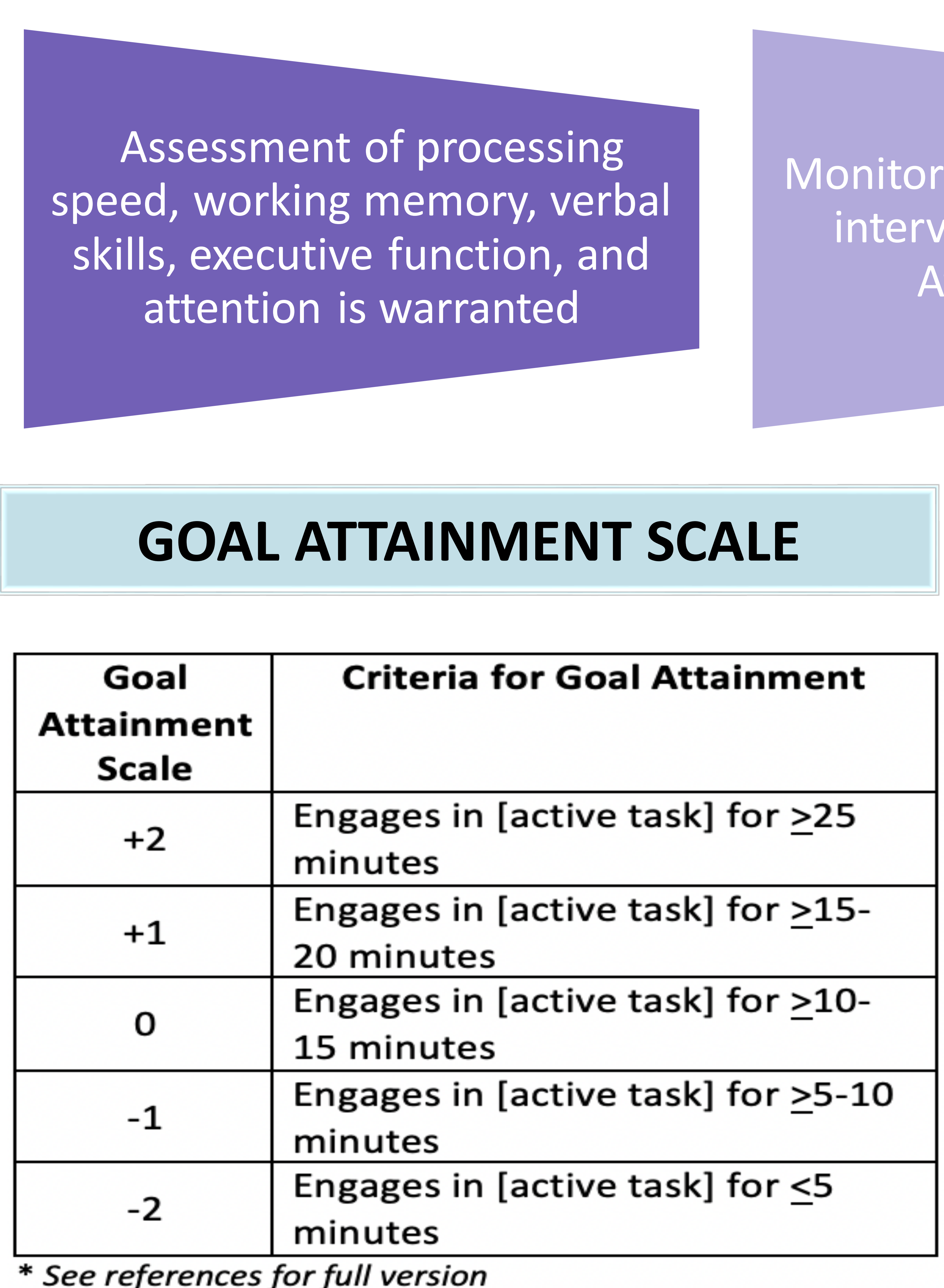

REFERENCES

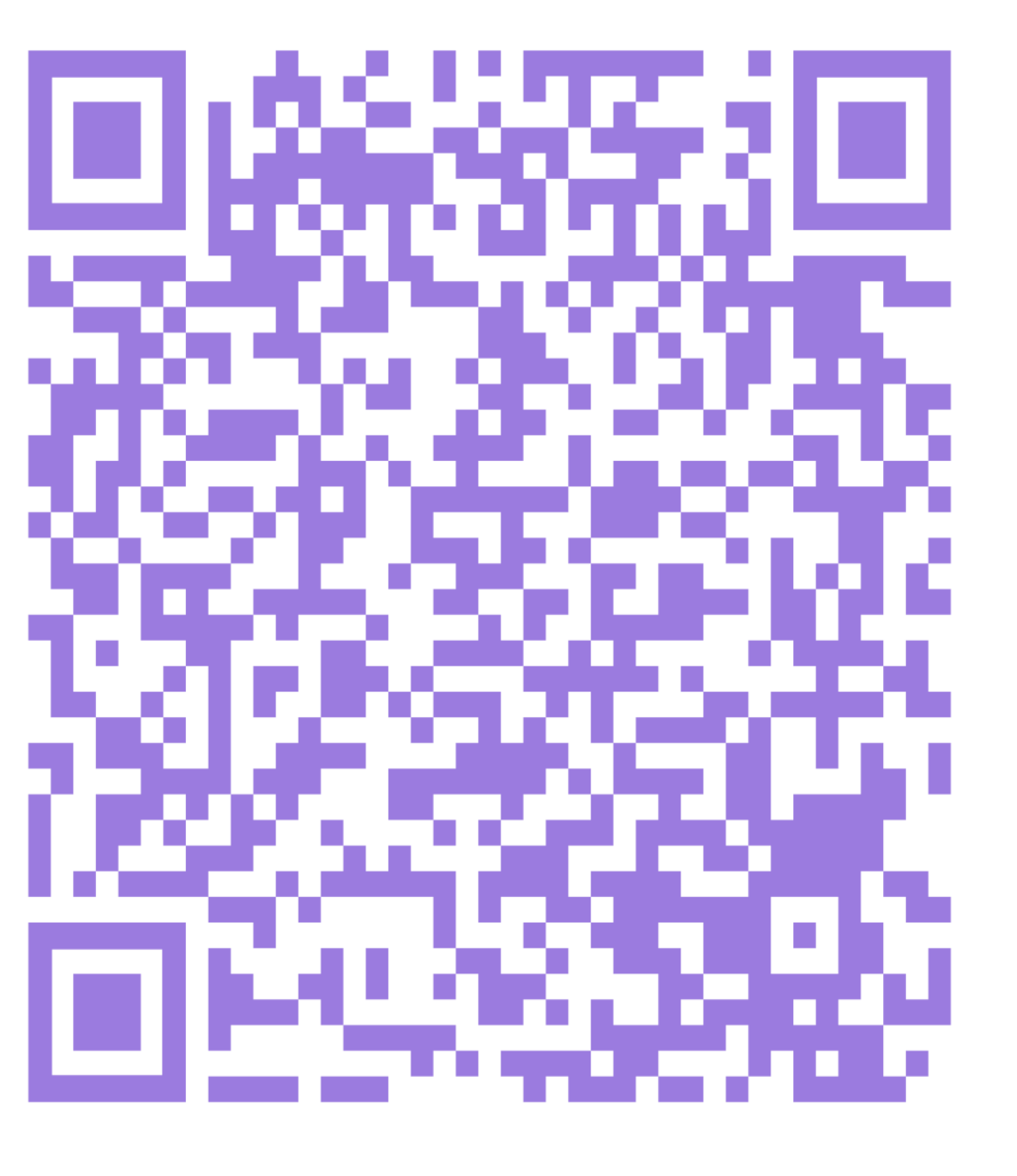

